\title{
Structure of PBP-A from Thermosynechococcus elongatus, a Penicillin- Binding Protein Closely Related to Class A $\beta$-Lactamases
}

\author{
Carole Urbach $^{1 \diamond}$, Christine Evrard ${ }^{2 \diamond}$, Vaidas Pudzaitis ${ }^{1}$, Jacques Fastrez ${ }^{1}$, Patrice Soumillion ${ }^{1}$ and Jean-Paul \\ Declercq $^{2}$ \\ ${ }^{1}$ Laboratoire d'Ingénierie des Protéines et des Peptides, Institut des Sciences de la Vie, Université catholique de Louvain, Place Croix du Sud \\ 4-5, bte3, 1348 Louvain la-Neuve, Belgium \\ ${ }^{2}$ Unit of Structural Chemistry (CSTR), Université catholique de Louvain, Place Louis Pasteur 1, 1348 Louvain-la-Neuve, Belgium
}

\begin{abstract}
Molecular evolution has always been a subject of discussions, and researchers are interested in understanding how proteins with similar scaffolds can catalyze different reactions. In the superfamily of serine penicillinrecognizing enzymes, D-alanyl- D-alanine peptidases and $\beta$-lacta-mases are phylogenetically linked but feature large differences of reactivity towards their respective substrates. In particular, while $\beta$-lactamases hydrolyze penicillins very fast, leading to their inactivation, these molecules inhibit D-alanyl-D-alanine peptidases by forming stable covalent penicilloyl enzymes. In cyanobacteria, we have discovered a new family of penicillinbinding proteins (PBPs) presenting all the sequence features of class A $\beta$-lactamases but having a six-amino-acid deletion in the conserved $\Omega$-loop and lacking the essential Glu166 known to be involved in the penicillin hydrolysis mechanism. With the aim of evolving a member of this family into a $\beta$-lactamase, PBP-A from Thermosynechococcus elongatus has been chosen because of its thermostability. Based on sequence alignments, introduction of a glutamate in position 158 of the shorter $\Omega$-loop afforded an enzyme with a 50-fold increase in the rate of penicillin hydrolysis. The crystal structures of PBP-A in the free and penicilloylated forms at $1.9 \AA$ resolution and of L158E mutant at $1.5 \AA$ resolution were also solved, giving insights in the catalytic mechanism of the proteins. Since all the active-site elements of PBP-A-L158E, including an essential water molecule, are almost perfectly superimposed with those of a class A $\beta$-lactamase such as TEM-1, the question why our mutant is still 5 orders of magnitude less active as a penicillinase remains and our results emphasize how far we are from understanding the secrets of enzymes. Based on the few minor differences between the active sites of PBP-A and TEM-1, mutations were introduced in the L158E enzyme, but while activities on D-Ala-D-Ala mimicking substrates were severely impaired, further improvement in penicillinase activity was unsuccessful.
\end{abstract}

Keywords: DD-peptidase; $\beta$-lactamase; PBP; carboxypeptidase; cyanobacteria

Abbreviations used: PBP, penicillin-binding protein; DD-peptidase, D-alanyl-D-alanine peptidase; PRE, penicillin-recognizing enzyme; PenG, penicillin G; PEG, polyethylene glycol; PDB, Protein Data Bank.

\section{INTRODUCTION}

Among 1 million sequences from 150 genomes, there are 850,000 protein sequences clustered into 50,000 families, each of which has the same structural domain composition. Eighty percent of domain sequences are assigned to only 5000 types of domain families. ${ }^{1}$ Hence, folds must have been used several times by divergent evolution or they must have independently arisen from different protein ancestors (convergent evolution) to provide the functional range represented through the protein universe. Proteins derived from a common ancestor have evolved through gene duplications, substitutions, insertions, and deletions, giving birth to families of homologous proteins that can catalyze the same reaction with different substrate specificities or catalyze different reactions although sharing similar mechanisms. ${ }^{2}$ The heterogeneity in substrate specificity or type of reaction catalyzed within a family of proteins proves the versatility of a fold, and researchers have challenged this versatility by using directed evolution strategies to generate enzymes endowed with new functionalities. Nevertheless, evolving new or promiscuous activities remains a great challenge especially when new catalytic mechanisms must appear.

\footnotetext{
${ }^{\diamond}$ C.U. and C.E. contributed equally to this work.
} 
The superfamily of the serine penicillin-recognizing enzymes (PREs), ${ }^{3}$ containing the D-alanyl-D-alanine peptidases (DD -peptidases) and $\beta$-lactamases, is a good illustration of these difficulties. DD -peptidases are bacterial cell wall enzymes involved in peptido-glycan synthesis mainly exhibiting carboxypeptidase and transpeptidase activities. $\beta$-Lactam antibiotics react with their catalytic serine and inhibit them by forming a stable acyl-enzyme adduct. Hence, DD-peptidases are also called PBPs (penicillin-binding proteins). ${ }^{4}$ To counteract these antibiotics, bacteria have developed defense mechanisms, the main one being the expression of $\beta$-lactamases that are capable of hydrolyzing penicillins rapidly. $\beta$-Lactamases and DD-peptidases share a similar two-step mechanism of acylation and deacylation, similar consensus sequences in the active site, and similar fold. This led to the suggestion that they are divergent descendants of an ancestral PBP Homology is, however, undetectable by pairwise sequence alignments. There are three classes of serine $\beta$-lactamases, $\mathrm{A}, \mathrm{C}$, and $\mathrm{D}$, with class B representing metalloen-zymes, and two classes of PBPs each subdivided into three groups, and each class of $\beta$-lactamase is probably related to a different class of PBP. ${ }^{5}$

In class A $\beta$-lactamases, four consensus active-site motifs are found. Following Ambler numbering, ${ }^{6}$ the first three motifs are $S_{70} X X K_{73}$ containing the catalytic serine, $S_{130} D N_{132}$, and $K_{234} T / S_{236}$, whose roles in the catalytic mechanism have been reviewed. These motifs are also present in the other proteins of the PRE family, but in class A $\beta$-lactamases, a fourth $\mathrm{E}_{166} \mathrm{XXXN}_{170}$ motif is found, located on a conserved $\Omega$-loop. This loop comprises residues 164 to 179 and flanks the active site. It contains a strictly conserved glutamic acid (Glu166) essential for catalysis of acyl-enzyme deacylation but whose role in acylation is still controversial ${ }^{9,10}$ Though class A $\beta$-lactamases are the most widespread and studied, their catalytic mechanism remains incompletely understood, and the possible milestone events of their evolutive genesis from an ancestral PBP are even more mysterious.

We have recently discovered the existence of a new family of PBPs from cyanobacteria, which are homologous to class A $\beta$-lactamases and their closer PBP parents known today. Of these, PBP-A from Thermosynechococcus elongatus shares, on average, $28 \%$ identity with class A $\beta$-lactamases but no detectable sequence homology to known DD-peptidases. PBP-A showed high activity towards thioles-ters mimicking natural D-alanyl-D-alanine substrates, suggesting a DD-carboxypeptidase activity. Moreover, the enzyme was sensitive to $\beta$-lactams with kinetic parameters typical of a PBP and a similar two-pathway decomposition mechanism of the penicil-loylated enzyme. PBP-A features an $\Omega$-loop six residues shorter than that of $\beta$-lactamases and in which a leucine is aligned with the essential Glu166. The L158E mutation, however, did not generate an active $\beta$-lactamase since, as shown in Table 1, the penicillin turnover rate of the L158E mutant is improved by only a factor of $50 .{ }^{11}$

In this work, we have solved the crystal structures of PBP-A and the L158E mutant. Fine analysis of the structures allowed us to compare the PBP-A catalytic site with the closest members of the superfamily, clearly identifying a typical class A $\beta$-lactamase catalytic site and overall fold. It also suggested mutating some residues susceptible to be responsible for some disturbance in the catalytic site but these attempts to engineer a $\beta$ lactamase activity were not successful. Hence, this study is a good example of the difficulty of engineering new and efficient enzymatic catalysis starting from an appropriate fold but a weak promiscuous activity.

Table 1. Kinetic parameters of PBP-A and L158E mutant for hydrolysis of benzylpenicillin at room temperature

\begin{tabular}{|l|l|l|}
\hline & $k_{\mathrm{cat}} / \mathrm{K}_{\mathrm{m}}\left(\mathrm{M}^{-1} \mathrm{~s}^{-1}\right)^{\mathrm{a}}$ & $k_{\text {cat }}\left(\mathrm{s}^{-1}\right)^{\mathrm{b}}$ \\
\hline PBP-A & $1.1 \times 10^{4}$ & $2.7 \times 10^{-4}$ \\
\hline PBP-A-L ${ }_{158} \mathrm{E}$ & $1.6 \times 10^{3}$ & $1.4 \times 10^{-2}$ \\
\hline TEM-1 $^{\mathrm{c}}$ & $8.4 \times 10^{7}$ & 1500 \\
\hline
\end{tabular}

${ }^{\mathrm{a}} k_{\text {cat }} / \mathrm{K}_{\mathrm{m}}$ values are equal to $k_{2} / \mathrm{K}_{\mathrm{s}}$ constants and were calculated from time courses of acylation of $\left[{ }^{14} \mathrm{C}\right]-P e n G$ (see Supplementary Materials)

${ }^{\mathrm{b}} k_{\mathrm{cat}}$, values were measured spectrophotometrically with PenG at $232 \mathrm{~nm}$.

${ }^{\mathrm{c}}$ Constants for TEM-1 were taken from Ref. [52].

\section{RESULTS}

The open reading frame of PBP-A encodes a 368-amino-acid protein with a predicted N-terminal membrane anchor. For biochemical and structural analysis, it has been truncated of $92 \mathrm{~N}$-terminal amino acids, giving rise to a 277-amino-acid soluble protein. Throughout this article, residue numbering refers to the truncated protein for PBP-A and to Ambler numbering scheme for class A $\beta$-lactamases. ${ }^{6}$ Four crystal structures of PBP-A have been determined (PBP-A, PBP-A-L158E, and two penicilloylated forms named PBP-A-Pen and PBP-A-L158E- 
Pen, respectively) with 4 monomers in each asymmetric unit. The electron density is usually well defined except for some residues at the $\mathrm{N}$-terminal and the $\mathrm{C}$-terminal parts of the polypeptides. The details are summarized in Table 2.

The Ramachandran diagrams (not shown) produced by Procheck ${ }^{12}$ show that $90 \%$ of the residues are in the most favored regions. One residue (Ile184) in the four independent molecules of the four structures systematically lies in a region $\left(\varphi \sim 75^{\circ}, \psi \sim-55^{\circ}\right)$ classified as disallowed by Procheck. However, according to the new Ramachandran criteria, ${ }^{13}$ this region is now classed as allowed and corresponds to a $\gamma$-turn. The 16 monomers are very similar, with a root-mean-square deviation (r.m.s.d.) of 0.427 A between the $C^{\alpha}$ of residues 11-274 observed in all of them. The largest discrepancy occurs at the level of residue 224, but this difference is induced by the packing, because in each of the four structures, only molecule $\mathrm{C}$ is concerned. Further similarity of the 16 molecules is their solvent-accessible areas with an average of 10,886 $\pm 77 \AA^{2}$ for PBP-A structures and 10,940 $\pm 118 \AA^{2}$ for PBP-A-L158E molecules. Despite the high level of similarity, local structural differences are observed, which may be explained by a different exposition of monomer of one structure to the crystal packing interactions.

The crystals are orthorhombic and belong to space group $\mathrm{P} 2{ }_{1} 2_{1} 2_{1}$ with a solvent content of $46 \%$. For each structure, four chains (A, B, C, and D), related by a 4-fold noncrystallographic symmetry axis along $c$, were observed in the asymmetric unit. Structured chain segments were variable for each monomer: for chains A, B, and $\mathrm{C}$ of all four structures, electron densities were observed between residue 10 or 11 and residue 274 or 275 with one exception for PBP-A chain C (residues 6 to 275); for chain D, the structured segments were longer going from residue 1 or 2 to residue 274,275 , or 276.

Table 2. Statistics of data collection and refinement

\begin{tabular}{|c|c|c|c|c|}
\hline & PBP-A & PBP-A-pen & $\mathrm{L}_{158} \mathrm{E}$ & $\mathrm{L}_{158}$ E-pen \\
\hline \multicolumn{5}{|l|}{ Data collection } \\
\hline Cell dimensions: $a / b / c(\AA)$ & \begin{tabular}{|l|}
$87.79 / 91.90 / 147$ \\
27
\end{tabular} & $\begin{array}{l}87.69 / 91.87 / 147 \\
44\end{array}$ & $\begin{array}{l}86.61 / 91.46 / 145 \\
59\end{array}$ & $\begin{array}{l}86.58 / 91.53 / 145 \\
.71\end{array}$ \\
\hline Resolution overall (ov) ( $(\AA)$ & $39.2-1.9$ & $24-1.9$ & $25-1.5$ & $28-1.7$ \\
\hline Resolution highest shell (hs) $(\AA)$ & $1.95-1.90$ & $1.95-1.90$ & $1.54-1.50$ & $1.74-1.70$ \\
\hline Unique reflections (redundancy) & $94,191(5.1)$ & $91,292(3.3)$ & $181,250(3.4)$ & $126,734(3.3)$ \\
\hline Completeness $(\%)$ ov/hs & $99.8 / 99.7$ & $96.7 / 88.2$ & $98.1 / 99.2$ & $99.3 / 100.0$ \\
\hline$R_{\mathrm{sym}}$ & 0.079 & 0.053 & 0.036 & 0.040 \\
\hline$I / \sigma(I)$ ov/hs & $13.9 / 3.8$ & $17.3 / 2.6$ & $17.6 / 2.3$ & $16.8 / 2.6$ \\
\hline \multicolumn{5}{|l|}{ Refinement } \\
\hline \multicolumn{5}{|l|}{ R-factor $\left(\mathrm{R}_{\text {free }}\right)$} \\
\hline ov & $0.174(0.213)$ & $0.190(0.245)$ & $0.179(0.213)$ & $0.180(0.218)$ \\
\hline hs & $0.220(0.298)$ & $0.273(0.346)$ & $0.245(0.273)$ & $0.242(0.285)$ \\
\hline \multicolumn{5}{|l|}{ Estimated overall coordinate error $(\AA)$} \\
\hline Based on $R_{\text {free }}$ & 0.132 & 0.156 & 0.078 & 0.104 \\
\hline Based on maximum likelihood & 0.097 & 0.118 & 0.057 & 0.079 \\
\hline r.m.s.d. from ideality: bonds $(\AA) /$ angles $\left({ }^{\circ}\right)$ & $0.015 / 1.51$ & $0.016 / 1.58$ & $0.017 / 1.646$ & $0.015 / 1.555$ \\
\hline Number of water molecules & 870 & 823 & 1527 & 905 \\
\hline Number of glycerol molecules & - & - & 3 & 1 \\
\hline $\begin{array}{l}\text { Mean B values }(\AA) \text { : main chain/side } \\
\text { chain/solvent }\end{array}$ & $26.8 / 29.1 / 35.4$ & $25.5 / 27.7 / 35.6$ & $23.4 / 26.2 / 40.5$ & $28.0 / 31.0 / 38.5$ \\
\hline
\end{tabular}


Fig. 1. Superposition of PBP-A molecule A (green) and TEM-1 $\beta$-lactamase (red).

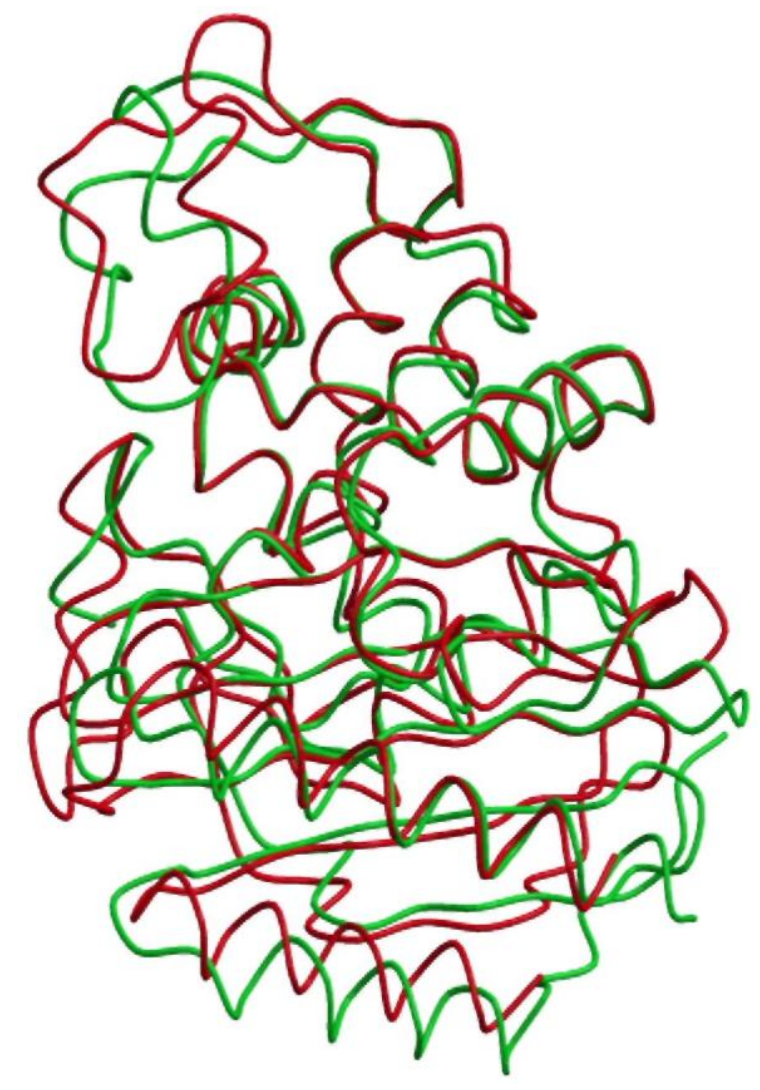

\section{The fold of PBP-A}

The PBP-A fold is very similar to the general fold of serine PREs. It is composed of two domains: an all $\alpha$ domain consisting of an array of five helices from residues 58 to 199 and an $\alpha \beta$-domain from residues 18 to 54 and 204 to 274, consisting of a six-stranded antiparallel $\beta$-sheet, packed on one side against C-terminal helix $\alpha 8$ and on the other side on helix $\alpha 6$, the active site being at the interface between the two domains. Two hinges connecting the domains can be defined from residues 54-58 and 200-204. The N-terminal part is only observed in molecule D of each structure and consists of a completely unstructured arm. When submitted to DALI server, ${ }^{14}$ the best superimposed structures were class A $\beta$-lactamases (PER-1 from Pseudomonas aeruginosa and TEM-1 from Escherichia coli) followed by several PBPs among which are PBP-5 from E. coli, PBP-4 from Staphylococcus aureus, PBP-4a from Bacillus subtilis, Streptomyces K15 peptidase, and PBP-1a from Streptococcus pneumoniae. The similarity of folds of PBP-A and TEM-1 is displayed in Fig. 1. As shown in Table 3, superposition with class A $\beta$-lactamases is better than that with PBPs. This is also true for the structural alignment of PER-1, TEM-1, PBP5, and PBP-A (Fig. 2).

Table 3. r.m.s.d. between PBP-A, penicillin-binding domain of PBP5, and two class A $\beta$-lactamases

\begin{tabular}{|c|c|c|c|c|}
\hline & & TEM-1 (1btl) & Per-1 (1e25) & PBP5 (1z6f) \\
\hline$\overline{\text { PBP-A }}$ & $\begin{array}{l}\text { r.m.s.d }(\AA) \\
\text { Number of aligned } C^{\alpha}\end{array}$ & $\begin{array}{l}1.83 \\
234\end{array}$ & $\begin{array}{l}1.58 \\
240\end{array}$ & $\begin{array}{l}2.27 \\
198\end{array}$ \\
\hline Per-1 & $\begin{array}{l}\text { r.m.s.d }(\AA) \\
\text { Number of aligned } C^{\alpha}\end{array}$ & $\begin{array}{l}1.75 \\
245\end{array}$ & - & $\begin{array}{l}\mathrm{ND} \\
-\end{array}$ \\
\hline PBP5 & $\begin{array}{l}\text { r.m.s.d }(\AA) \\
\text { Number of aligned } C^{\alpha}\end{array}$ & $\begin{array}{l}2.62 \\
212\end{array}$ & ND & - \\
\hline
\end{tabular}


Fig. 2. Structural alignment of TEM-1 (1btl), PBP-A chain A, PBP5, and PER-1. Structural alignment was achieved with Mustang ${ }^{54}$ and edited using BioEdit. ${ }^{55}$ Residue numbering corresponds to that of TEM-1; gaps are indicated in red. Magenta boxes are SXXK, SXN, and KT/SG motifs, respectively. Purple box is the $\Omega$-loop region, and Glul66 is highlighted in magenta.

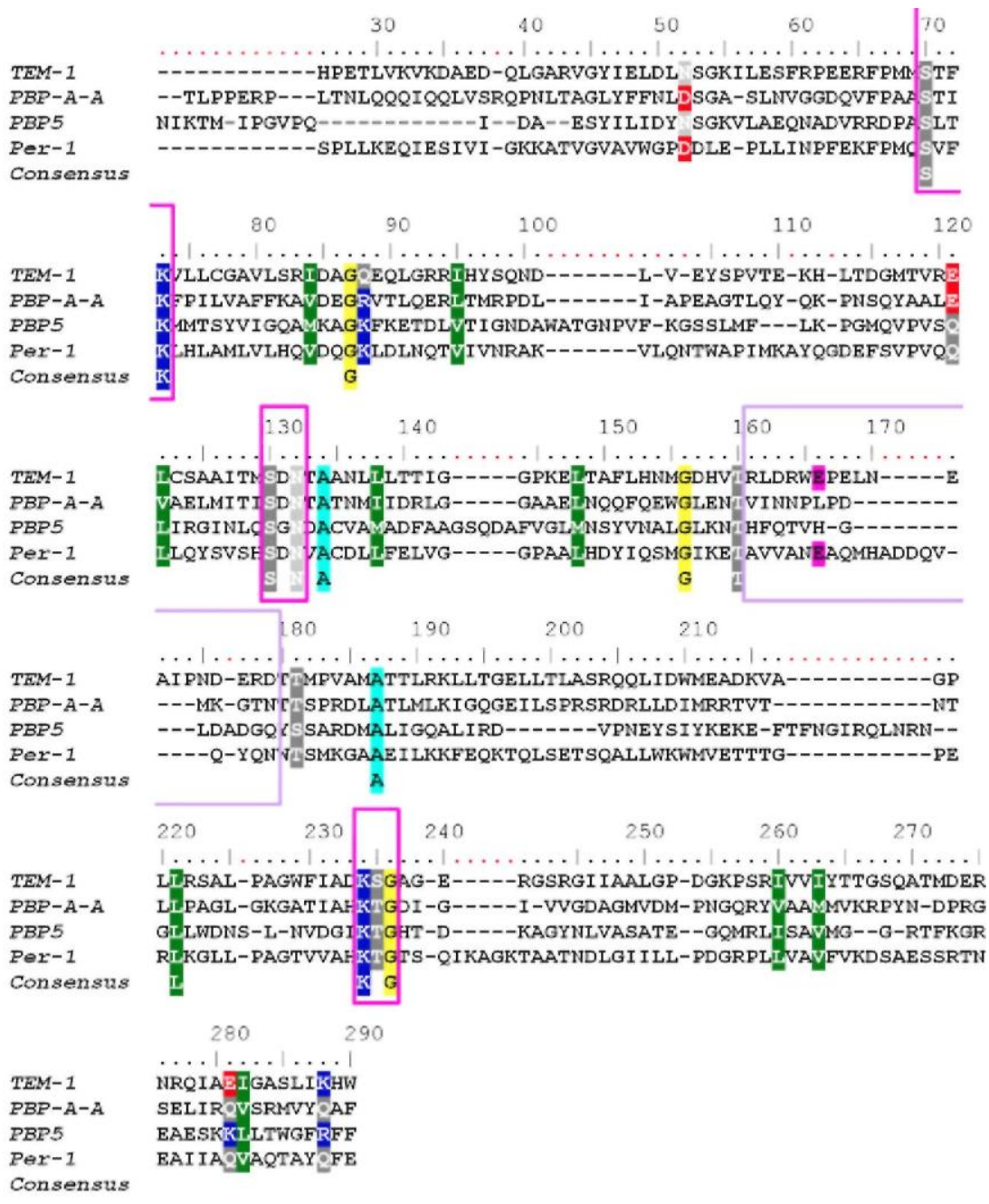

\section{Active site}

The active site was located by plotting the conserved sequence motifs on the structure. The entrance to the active site is at the opposite side of the putative $\mathrm{N}$-terminal membrane anchor, in an ideal position to interact with cell wall peptides. Within PBP-A, the essential serine 61 and lysine 64 are located at the N-terminal end of helix $\alpha 2$, the turn between helices $\alpha 3$ and $\alpha 4$ contains Ser122 and Asn 124 of the second conserved motif, and Lys 219 , Thr220, and Gly221 are situated at the edge strand of the $\beta$-sheet. Similarly to what is observed in PREs, the PBP-A active site shows a dense hydrogen-bonding network conserved in all the structures and residues in the active site are involved in many interactions (Fig. 3). In common with many other PRPs, the $\zeta-\mathrm{NH}_{2}$ group of Lys64 plays a pivotal role in this network, forming hydrogen bonds with the hydroxyl group of Ser61 (2.78 $\AA$ ), the hydroxyl and backbone carbonyl groups of Ser122 (3.08 and $2.78 \AA$ ), and the amide carbonyl group of Asnl24 (2.79 $\AA$ ). The $\zeta-\mathrm{NH}_{2}$ group of Lys219 forms a hydrogen bond with the hydroxyl group of Ser122 (2.83

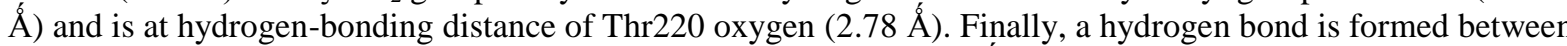
the Ser61 hydroxyl group $\mathrm{O}^{\gamma}$ and the Ser122 hydroxyl group $\mathrm{O}^{\gamma}(3.02 \AA)$ ). For class A $\beta$-lactamases, distances of $3.17 \AA$ for PER-1 (1e25), ${ }^{15} 3.15$ A for TEM-1 in $1.55 \AA$ A resolution structure $(1 \mathrm{zg} 4),{ }^{16}$ but $3.55 \AA$ in $1.8 \AA$ resolution structure (1btl), ${ }^{17}$ and $2.89 \AA$ in Toho- 1 (1iys) ${ }^{18}$ are found. In class A $\beta$-lactamases, the $\mathrm{Lys} 73 \zeta-\mathrm{NH}_{2}$ group is hydrogen bonded to the carboxylate group of Glu166. This is not the case in the L158E mutant, although Glu158 is in a position similar to Glul66 in $\beta$-lactamases. 
In class A $\beta$-lactamases, two molecules of water are structurally conserved. ${ }^{19-21}$ In PBP-A and PBP-A-L158E, respectively, three and four molecules of water are observed in the active site (Fig. 3). The first one (Wl) is observed in both wild-type and mutant proteins where it occupies the oxyanion hole, a substructure that stabilizes the tetrahedral intermediate first described for class A $\beta$-lactamases by Murphy and Pratt and made up of the main-chain NH groups of Ser70 and residue $237 .{ }^{22} \mathrm{~W} 1$ is found at 3.06 and 3.40 Arom the equivalent Ser61 and Asp222 nitrogens for PBP-A. The distance between W1 and Asp222 suggests a weak electrostatic stabilization, whereas it forms a real hydrogen bond with the Ser61 nitrogen. Since the oxyanion hole is electrostatically positive, the negative charge of Asp222 may induce a weak destabilization. In TEM-1, the distances between main-chain nitrogen atoms of Ser70 and Ala237 and the water molecule are 2.94 and $3.16 \AA$, respectively, and in PER-1, the distances are $3.2 \AA$ between water and Ser70 and $2.8 \AA$ between water and Thr237.

The second water molecule conserved in the active site of class A $\beta$-lactamases is involved in penicillin hydrolysis and found at hydrogen bond distance of hydroxyl group of Ser70 and ع1-O of Glu166 carboxylate group. A similar water molecule, W2, is found in PBP-A-L158E mutant at 2.81 and $3.31 \AA$ of Glu158 and Ser61, respectively. The corresponding distances are 2.63 and $2.75 \AA$ in TEM- 1 and 2.86 and $2.98 \AA$ in PER-1 $\beta$ lactamases; this water molecule is also hydrogen bonded to the side-chain oxygen of Asnl70 at $3.23 \AA$ for TEM1 (1btl). In PBP-A-L158E, W2 is at hydrogen bond distance of the oxygen of Pro159 (2.79 A). In molecule D, W2 is slightly farther away compared to the others, at 3.51 and $3.65 \AA$ of Glu158 and Ser61, respectively (these distances were not included in the calculation of average distances). In the wild-type protein, a similar water molecule is found at $2.85 \AA$ distance from Pro $159 \mathrm{O}$, but the distance from W2 to Ser61 hydroxyl group is longer $(3.59 \AA)$ in molecules A, B, and C and even longer in molecule D (5.71 $\AA$ ). A third conserved water molecule, W3, is observed in the catalytic site of PBP-A. It is hydrogen bonded to the side chain of Lys219. This water molecule is found in all penicilloyl serine transferases and plays a structural role in stabilizing the side chain of the lysine of the KT/SG motif. ${ }^{23}$ The L158E mutation appears to bring another water molecule in the active site, at hydrogen bond distance of $\varepsilon 2-\mathrm{O}$ of Glu158 carboxylate group and Ala60 oxygen (not indicated in Fig. 3).

\section{Structure of covalent adduct}

In PBP-A-Pen, the acylation of Ser61 and the cleavage of the $\beta$-lactam ring between $\mathrm{N}_{4}$ and $\mathrm{C}_{7}$ are clearly observed in the four molecules of the asymmetric unit (Fig. 4). The situation is much less clear for PBP-AL158E-Pen. Some electron density appears in the catalytic pocket but the penicilloyl intermediate cannot be explicitly identified in this electron density, likely due to a low occupancy and a large motion. Because of this problem, probably related to the increased hydrolytic activity, this molecule will not be further discussed.

There is no striking structural difference between free PBP-A and acyl-enzyme, as evidenced by the r.m.s.d. of the eight structures: $0.425 \AA$ for $C^{\alpha}$ and $0.828 \AA$ for all atoms. For the active-site residues (S61-K64, S122-N124, and K219-D222), the r.m.s.d. is $0.206 \AA$ for all atoms, indicating that binding of the substrate has little effect on the active-site cavity. A difference is again observed in molecule D where the orientation of the penicillin phenyl group is very different (Fig. 6c).

A comparison of the acylated forms of PBP-A and of the TEM-1 E166N mutant (1fqg) ${ }^{21}$ is shown in Fig. 5, where the many hydrogen-bonding interactions stabilizing the acyl moiety are shown. There are few differences between the two structures. In both, the carbonyl oxygen atom $\mathrm{O}_{8}$ of the acyl moiety replaces the water molecule $\mathrm{W} 1$ in the oxyanion hole. The water molecule W2 present in the free PBP-A (not shown) still forms a strong

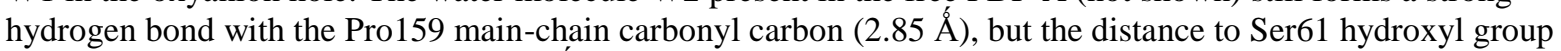
$\mathrm{O}^{\gamma}$ has increased in all molecules $(5.15 \AA \hat{)})$, whereas it is at the same place in PBP-A-L158E, indicating the importance of a negative side chain to maintain W2 in place for deacylation. In PBP-A, the penicillin G (PenG) carboxylate oxygen atom $\mathrm{O}_{12}$ forms a strong hydrogen bond to the hydroxyl group oxygen of Thr220 and a weak one to $\zeta-\mathrm{NH}_{2}$ group of Lys219, while $\mathrm{O}_{13}$ is only electrostatically stabilized by the same Thr220 hydroxyl group. In TEM-1 E166N, the carboxylate $\mathrm{O}_{12}$ forms a strong hydrogen bond with the hydroxyl of Ser235 while its interaction with the $\zeta-\mathrm{NH}_{2}$ of Lys224 is weaker but $\mathrm{O}_{13}$ is involved in a strong electrostatic interaction with the $\gamma$ $\mathrm{NH} 2$ of Arg244, which is absent and replaced by a valine in PBP-A. 
Fig. 3. Hydrogen bond network in the active site of PBP-A (a) and PBP-A-L158E (b). The segment of helix abearing motif SXXK is yellow, the segment of loop bearing motif SDN is green, the segment of $\Omega$-loop bearing $L / E 158$ is red, and the segment of strand $\beta 3$ bearing motif KTG is cyan. Hydrogen bonds are indicated by broken lines. Hydrogen bonds between water molecule Wl and main-chain nitrogen atoms of S61 and D222 are not indicated for clarity.

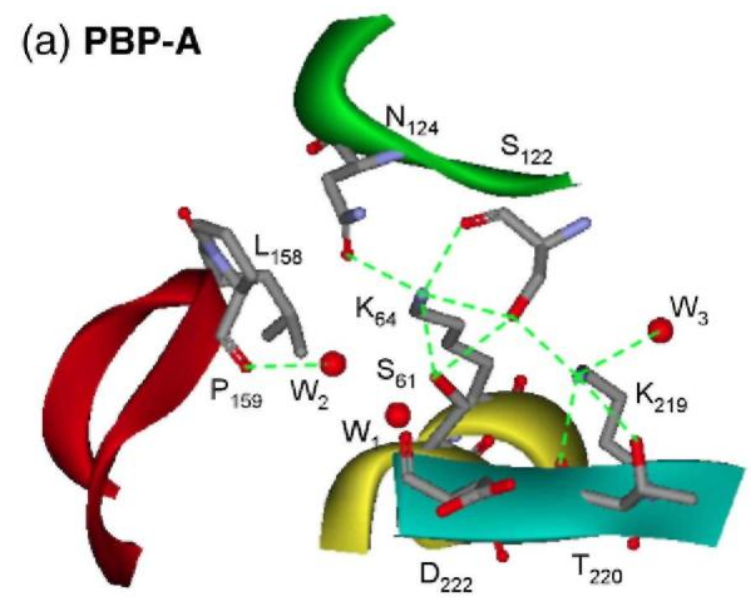

(b) PBP-A L158E

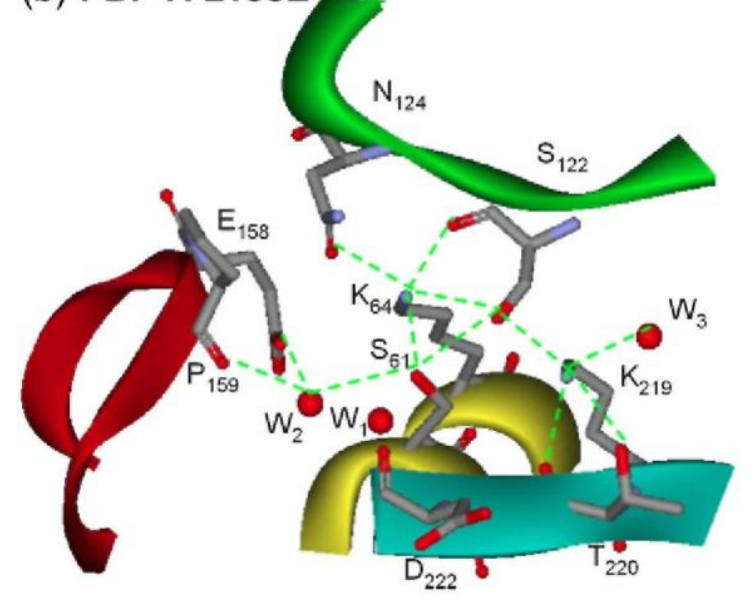


Fig. 4. Stereoview of the active site of PBP-A acylated by benzylpenicillin. The $\left(F_{o} F_{c}\right)$ omit electron density for the bound ligand is contoured at a level of $2.0 \sigma$.

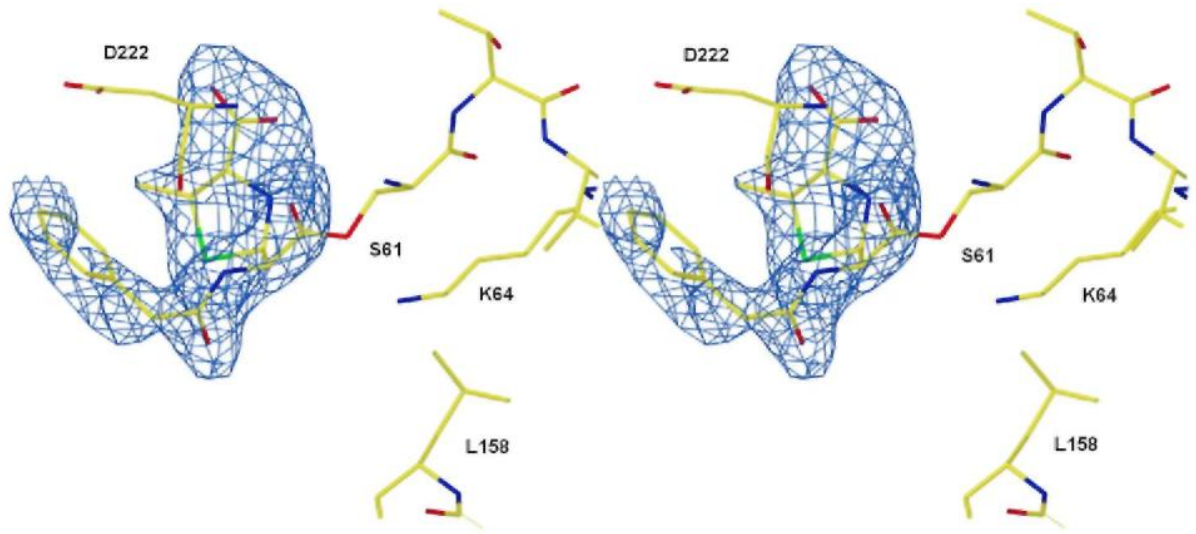

\section{Comparison with TEM-1 and PER-1 $\beta$-lactamases}

The spatial arrangement of the conserved residues in the active site is remarkably well conserved, and r.m.s.d. for the superposition of all atoms of active-site residues S70-K73, S130-N132, and K234-A237 and water molecules 1 and 2 in $\beta$-lactamases and equivalent amino acids and water molecules in PBP-A mutant is $0.914 \AA$ for TEM-1 (lzg4) and $0.807 \AA$ for PER-1 (1e25). Leu158, which, on the basis of sequence alignments, is aligned with Glu166, is also structurally at the same spatial position, and the Glu158 side chain in the mutant has the same orientation as in TEM-1 or PER-1.

Although the globular domain of PBP-A and that of TEM-1 share a very similar fold and active site, several significant differences should be pointed out. The most striking one is the $\Omega$-loop-like structure whose length is strictly conserved in natural class A $\beta$-lactamases but six amino acids shorter in PBP-A. Nevertheless, while there is no residue equivalent to Glu166 in PBP-A, its $\Omega$-loop-like structure fits rather well with the N-terminal end of TEM-1 $\Omega$-loop (Fig. 6a). In particular, the cis peptide bond between residues 166 and 167, an invariant feature of class A $\beta$-lactamases, except in PER-1, which displays a different $\Omega$-loop fold, ${ }^{15}$ is observed in position 159 of PBP-A. On the other hand, three invariable electrostatic interactions maintain the loop integrity in class A $\beta$-lactamases: a hydrogen bond between the side chains of Lys73 and Glu166, a salt bridge between Arg164 and Asp179, and hydrogen bonds between the CO and NH2 groups of Asn136 side chain and the mainchain NH and CO groups of Glu166, respectively. In PBP-A, there are many strong hydrogen bonds maintaining the loop structure but no salt bridge. The Asn136-Glu166 interactions remain between the equivalent residues Asn128 and Leu/Glu158 in PBP-A and PBP-A-L158E. Consequently, the $\Omega$-loop appears to be able to put Glu158 in a position adequate for water activation, although it may not be optimal. Indeed, when the TEM-1 and PBP-A active sites are superimposed, the $C^{\delta}$ of Glu166 and Glul58 are separated by $1.63 \AA$ A. In class A $\beta$ lactamases, the hydrolytic water molecule, W2, is hydrogen bonded to Glu166 and Asn170. This residue seems to be important for the hydrolytic activity since its mutation impairs the enzyme activity. ${ }^{24-26}$ In PBP-A-L158E, it is the carbonyl oxygen of Pro159 that binds W2, which remains at 3.31 Á from hydroxyl group of Ser61. This is a little far when compared to $\beta$-lactamases with $2.75 \AA$ for TEM- 1 and $2.98 \AA$ for PER-1.

Another difference concerns the loop connecting helices $\alpha 2$ and $\alpha 3$ (residues 91-106) and overhanging the catalytic site. Its conformation is different from that of residues 100-115 of the class A $\beta$-lactamases.

Particularly, residues 104 and 105 of TEM-1, which play a role in substrate binding and specificity ${ }^{27-29}$ and indirectly in catalysis, ${ }^{30-31}$ are very badly aligned with the equivalent residues Glu96 and Ala97 of PBP-A and there is an amino acid insertion (Fig. 6b). In class A $\beta$-lactamases, except in PER-1, residue 104 is hydrogen bonded to the side chain of the essential Asn132 through the backbone carbonyl oxygen. In PBP-A (except in molecule D), the carbonyl oxygen of Ala97 is hydrogen bonded to the $\delta-\mathrm{NH}_{2}$ amide group (3.20 $\AA$ ) and the main-chain nitrogen (2.99 $\AA$ ) of Asnl24. Hence, the stabilizing role of this loop seems to be maintained, even though the global conformation is different. This loop and particularly Tyr105 in TEM-1 contribute also to the enzyme specificity ${ }^{29}$ by forming a wall in contact with the substrate, but an aromatic amino acid is not necessary and small residues such as alanine and glycine that do not disturb the structure of the active site are allowed. In PBP-A, the residue aligned with Tyr105, Ala97, does not seem to disturb the structure of the cavity, but the 
conformation of the side chain of Glu96 is different from that of Glu104, and it brings a negative charge that closes the left side of the cavity, which may be a problem for penicillin binding. Indeed, different penicillin conformations are observed in the cavities of TEM-1, Streptomyces R61 DD-peptidase, and PBP-A. Whereas the substrate side chain is oriented similarly in the R61 and TEM catalytic site, it appears more distorted in PBP-A, certainly under the influence of Glu96 (Fig. 6c).

A deletion is observed in one of the two hinges connecting the helical domain to the $\alpha \beta$-domain: there is no equivalent to TEM-1 Ala 217 in PBP-A. Though this region is important for domain association and thus for the good positioning of catalytic apparatus, there is little residue conservation in this loop among class A $\beta$ lactamases. ${ }^{32}$ Moreover, circularly permuted lactamases at position $228^{33}$ or 216 retained enough activity to confer some in vivo ampicillin resistance. Hence, a deletion in this region may not be of great importance.

The last region that undergoes minor differences when compared to class A $\beta$-lactamases is around the KTG motif. Before this motif, Asp233, conserved in most class A $\beta$-lactamases, is important for a correct active-site conformation. ${ }^{7}$ In PER-1, a histidine is found at this position and its interactions with residues Met211, Thr214, Thr216, and Asp246 appear to stabilize the hinge and contribute to align the two domains. In PBP-A, Met197, Thr200, Thr202, and Asp229 are spatially located in similar positions versus His218 although the detailed interactions with the threonines are different. Hence, a hydrogen bond network similar to that of PER-1 appears to be present in PBP-A, with the probable role of maintaining adequate interactions between the $\alpha$ - and $\alpha / \beta$ domains. In TEM-1, Asp246 and the threonines are not conserved, and the only similar interaction is the hydrogen bond with Met211. 
Fig. 5. Hydrogen bond network stabilizing the acyl-enzyme complex formed with benzylpenicillin and PBP-A (a) or TEM-1 E166N (b). Benzylpenicillin is black, residues from the enzymes are blue as well as hydrogen bonds, and red dot lines are weak hydrogen bonds.

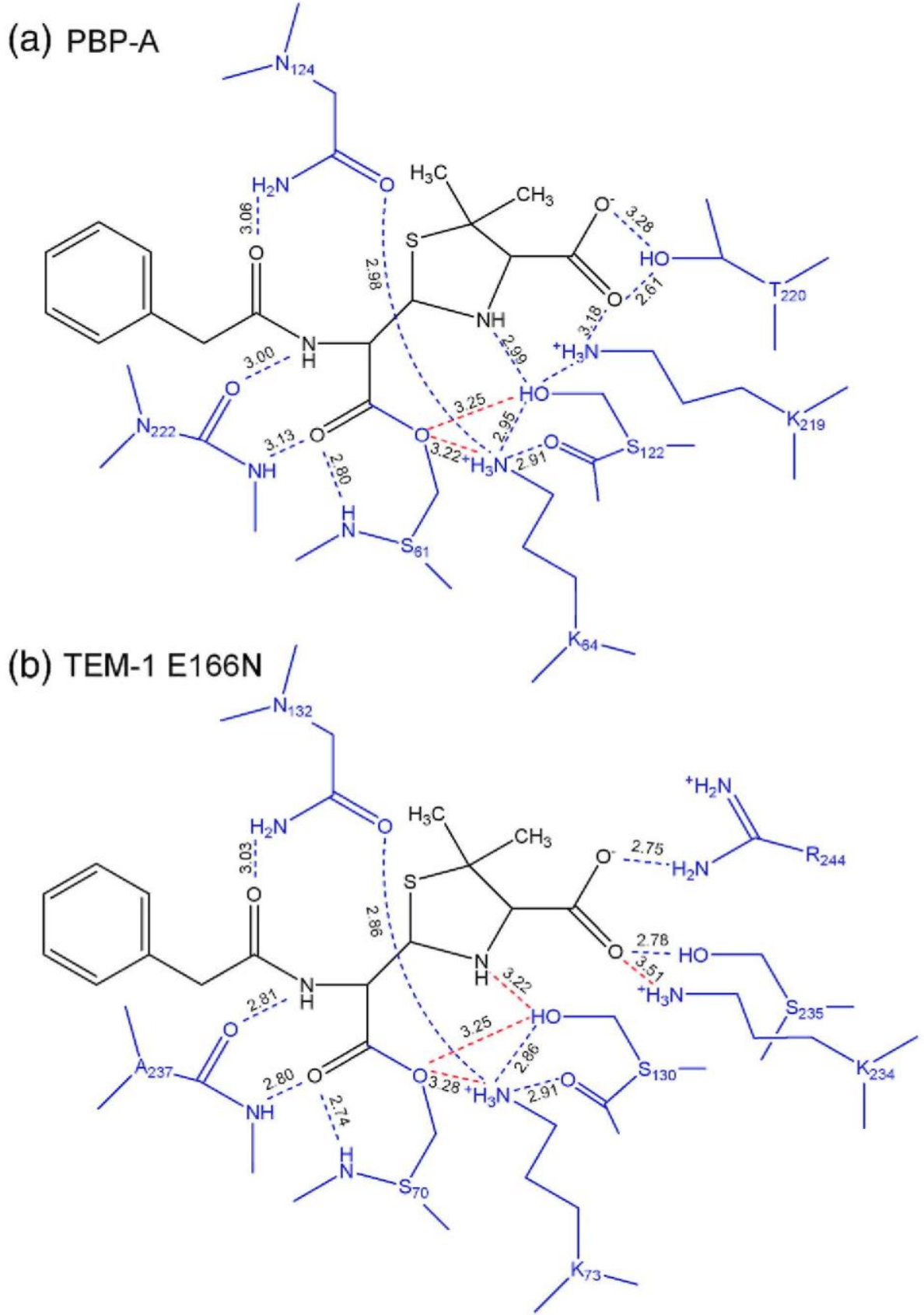


Fig. 6. (a) Superposition of PBP-A (green), PBP-A-L158E (cyan), and TEM-1 (red) in the region of the $\Omega$-loop. (b) Detailed view of the conformation of the 91-106 region in PBP-A (green) and TEM-1 (red), (c) Orientation of benzylpenicillin in the catalytic site of TEM-1 E166N mutant (green), R61 DD-peptidase (cyan), and PBP-A molecules $A$ (red) and D (pink). The PDB code for penicilloylated R61 is lpwc. ${ }^{56}$

(a)
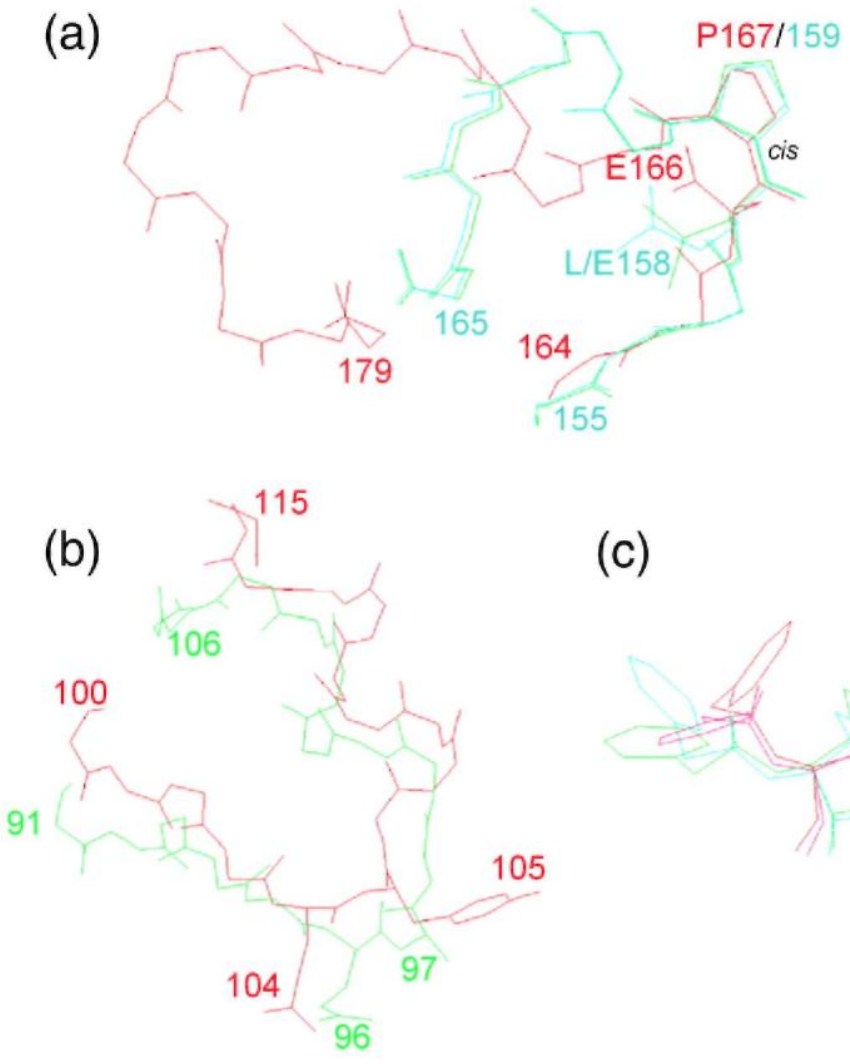

(c)

\section{Mutants}

The fact that the Leu158 mutation into a glutamic acid afforded only a modest improvement of the penicillinase activity suggested that the catalytic site needs to be finely tuned in order to better accommodate and hydrolyze $\beta$ lactam substrates. Hence, several additional mutations were considered.

Attempts were first made to position the hydro-lytic water molecule W2 closer to Ser61 and/or influence the Glu158 side-chain conformation by introduction of a longer polar residue in replacement of Pro159. Mutational modelling with Swiss PDBViewer ${ }^{35}$ indicates that, in the mutants D160N and M161N, the Asn could adopt conformations where it points towards the active site. Hence, four mutants were generated: PBP-A-D160N, PBPA-L158E-D160N, PBP-A-M161N, and PBP-A-L158E-M161N, with the single mutants being constructed as negative controls. The activities of the mutants on benzylpenicillin (PenG) were measured. The D160N mutation did not have any significant effect on activity when residue 158 was a leucine. The acylation rate was increased five times in PBP-A-L158E-D160N compared to PBP-A-L158E, but dea-cylation was impaired to reach the level of PBP-A. The M161N mutation led to a small increase in the rate of deacylation in PBP-A, which was not retained in the Glu158 mutant (Supplementary Materials, Table 4).

A second set of mutations aimed at improving the interactions of PBP-A with the penicillin carboxylate by introduction of a guanidinium group in its vicinity, a role played by Arg244 in TEM-1 ${ }^{36}$ and Arg220 in Streptomyces albus $G$ lactamase. ${ }^{37}$ The aligned residues, Val227 and Leu205, were individually mutated to arginine in PBP-A-L158E. The V227R mutation did not change the catalytic activity on PenG; L205R led to an inactive protein. 
Finally, attempts were made to correct a potential deleterious effect of negative charges on the rim of the active site. Glu96, in the loop connecting helices $\alpha 2$ and $\alpha 3$, was mutated to glycine or valine, a small hydrophobic residue. Asp222, in the loop between $\beta$-strands 3 and 4, which also contains one additional residue that may influence the local conformation in $\beta$-lactamases, was replaced by Ala-Gly. This replacement was combined with the E96G or E96V and L158E mutations. Both mutants, E96G-L158E-M161N-D222AG (E96G+) and E96V-L158E-M161N-D222AG (E96V+), showed a decreased penicillinase activity. Which of the E96 or D222 mutations was responsible for the decreased rate of deacylation was not assessed. It was surprising to see that PBP-A was able to support four mutations close to the active site without losing the activity for penicillin hydrolysis.

The effect of these mutations on the rate of hydrolysis of thiolesters mimicking the D-alanyl-D-aianine substrates was also measured (Supplementary Materials, Table 5). The L158E mutation had a deleterious effect, which could not be corrected by the additional mutations.

\section{DISCUSSION}

Biochemical characterization suggested that PBP-A is a DD-carboxypeptidase while sequence alignments indicated that it belongs to a new class of PBPs. ${ }^{11}$ The tridimensional structure of the protein reported here supports these hypotheses. On the other hand, structural alignments are better with class A $\beta$-lactamases than with other PBPs. Comparisons to other PBPs show less structural conservation, and though hydrophobic elements typical of PBPs seem to be present, they are organized differently. PBP-A is the first protein in which an $\Omega$-loop-like structure fits so well with the $\mathrm{N}$-terminal part of that of $\beta$-lactamases. In comparisons, many similarities were detected with TEM-1, but PBP-A shares also some similarities with the very special PER-1 $\beta$ lactamase. All these observations strengthen the theory of an ancestral PBP that would be an evolutionary intermediate between PBP-A and class A $\beta$-lactamases.

$\beta$-Lactamases and PBPs act on their substrates by a two-step mechanism involving a proton shuttle. In the first step, the enzyme is acylated by the substrate: the nucleophilic attack of the carbonyl carbon by the activated $\mathrm{O}^{\gamma}$ of the catalytic serine is concomitant with the transfer of a proton from the hydroxyl group of a conserved serine to the leaving group. In the second step, a proton from the acceptor (an ammonium group or a water molecule) is abstracted, the activated acceptor attacks the carbonyl carbon of the acyl-enzyme, and the abstracted proton is back-donated to $\mathrm{O}^{\gamma}$ of the leaving serine, hence deacylating the enzyme.

The rate of acylation of PBP-A by benzylpenicillin $\left(k_{\mathrm{Cat}} / \mathrm{Km} \approx 10^{4} \mathrm{M}^{-1} \mathrm{~s}^{-1}\right)$ is nearly 10,000 times slower than that of TEM-1 $\beta$-lactamase but not far from the maximum value observed with PBPs, which, despite a similar organization of their active site, show a wide variation in penicillin acylation efficiencies, ranging from $20 \mathrm{M}^{-1}$ $\mathrm{s}^{-1}$ for the low-affinity PBP5fm to $300,000 \mathrm{M}^{-1} \mathrm{~s}^{-1}$ for the best R39 PBP. For PBPs, the general base activating the nucleophilic serine must be the lysine of the SXXK motif. For $\beta$-lactamases, the identification of the general base has been the source of considerable controversy: ${ }^{21,38-41}$ both Glu166 acting through a water molecule or Lys 73 have been proposed. Mutation of Glu166 to Gln or Ala affords enzymes that can still be acylated by penicillins although at a much slower rate. This implies that both Glu166 and Lys73 are able to catalyze acylation. Recent simulations suggest the existence of two competing pathways but in which these residues can cooperate. $^{8,9}$ The PBP-A acylation efficiency is in an intermediate range probably because the shape and the electrostatic potential of the active-site cavity may not be optimal; in the L158E mutant, the interaction between Glu158 and Lys64 is lost.

The rate of deacylation of PBP-A and its L158E mutant is $5.5 \times 10^{6}$ and $1.1 \times 10^{5}$ times slower than that of TEM1. Glul66 is clearly the general base that activates the water molecule in class A $\beta$-lactamases since its mutation severely impairs the deacylation process in TEM-1. ${ }^{8,38}$ In some PBPs, a water molecule occupying a position similar to the TEM-1 deacylating water is sometimes present. However, there is no residue equivalent to Glu166 able to activate it, thus explaining the low deacylation rates. ${ }^{23,42}$ In the case of PBP2x, the aromatic ring of Phe450 hampers the entry of a water molecule in the active site. However, its mutation to an aspartic acid leads to only a 110 -fold increase in deacylation rate. The active site may not be fit to catalyze the hydrolysis since no water molecule is preexistent in the wild-type PBP2x catalytic site and no water molecule in its oxyanion hole. $^{43,44}$ The catalytic site of PBP-A, on the other hand, contains the two water molecules conserved in $\beta$ lactamases and is very similar to that of TEM-1. Furthermore, modeling of the tetrahedral intermediate of deacylation indicates that there is room in the L158E mutant for a deacylating water. Clearly, the fact that the introduction of a glutamate spatially in the same position as Glu166 does not improve the rate of deacylation more than 90 times remains puzzling since in the L158E mutant, all the "catalytic" residues and the interactions characteristic of $\beta$-lactamases seem to be present. This may result from the fact that the electrostatic environment 
or the hydrophobicity of the cavity does not provide a configuration suitable for the important proton shuttles involved in the mechanism. However, the adjustments of the shape or electrostatic potential of the cavity that we have tried to introduce did not allow modifying significantly the catalytic properties of the L158E mutant.

In conclusion, there is no obvious explanation why this protein is not a $\beta$-lactamase. Our results are, thus, a good illustration of how proteins are not as versatile as they look and that enzyme efficiencies are often mysteries for rational studies.

\section{MATERIALS AND METHODS}

Figures were achieved using Chimera $1.0^{45}$ and View-erLite 5.0 (Accelrys).

\section{Construction and purification of PBP-A and mutants}

Mutants were constructed using the QuickChange ${ }^{\circledR}$ Site-Directed or Multi-Site-Directed Mutagenesis Kit (Stratagene) (see Supplementary Materials for details). The mutations were confirmed by sequencing of both strands on the entire gene. The wt PBP-A and mutants were expressed in E. coli and purified as described previously ${ }^{11}$

\section{Kinetic measurements}

The parameter $k_{\text {cat }}$ for benzylpenicillin hydrolysis was determined by following absorbance at $232 \mathrm{~nm}$. The acylation constants $k_{2} / K_{s}\left(=k_{\text {cat }} / \mathrm{K}_{\mathrm{m}}\right)$ were determined from time courses of formation of acyl-enzyme complexes of $\left[{ }^{14} \mathrm{C}\right]-\mathrm{PenG}$ with the proteins, and the deacylation rate constant was derived from the first-order decay rates of the $\left[{ }^{14} \mathrm{C}\right]$ penicilloyl-PBP complex as described in Supplementary Materials.

\section{Crystallization}

The concentrations of the wild-type protein and of the $\mathrm{L}_{158} \mathrm{E}$ mutant used for crystallization experiments were about $7 \mathrm{mg} / \mathrm{mL}$, with $20 \mathrm{mM}$ Hepes, pH7.4, and ethylenediaminetetraacetic acid $1 \mathrm{mM}$. The hanging drop vapor diffusion method was used at $18^{\circ} \mathrm{C}$. For the wild-type protein, the reservoir was filled with $500 \mu \mathrm{L}$ of an aqueous solution containing 0.1 M Hepes, pH7.5,0.2 M proline, 10\% (w/v) polyethylene glycol (PEG) 3350, and $0.02 \%(\mathrm{w} / \mathrm{v}) \mathrm{NaN}_{3}$. The crystals used for acylation were identical but grown from a different reservoir composition: 0.1 M Hepes, pH7.5, 0.2 M ammonium acetate, 25\% (w/v) PEG 3350, and 0.02\% (w/v) $\mathrm{NaN}_{3}$. For the $\mathrm{L}_{158} \mathrm{E}$ mutant, the reservoir solution contained $0.1 \mathrm{M}$ Hepes, pH6.9, 0.2 M ammonium acetate, $15 \%(\mathrm{w} / \mathrm{v})$ PEG 3350, and $0.02 \%(\mathrm{w} / \mathrm{v}) \mathrm{NaN}_{3}$. The hanging drop was composed of $1 \mu \mathrm{L}$ of protein solution mixed with 1 $\mu \mathrm{L}$ of the reservoir. In both cases, crystals with typical dimensions of $0.15 \mathrm{~mm} \times 0.15 \mathrm{~mm} \times 0.10 \mathrm{~mm}$ appeared after a few days.

\section{Data collection}

Before data collection, the crystals were soaked for a few seconds in a cryosolution similar to the mother liquor but containing $5 \%$ glycerol as cryoprotectant and flash cooled at $100 \mathrm{~K}$. To study the acyl-enzymes, we also soaked some crystals for $1 \mathrm{~min}$ in such a solution containing PenG. All the diffraction data were collected using synchrotron radiation at the European Molecular Biology Laboratory c/o Deutsches Elektronen-Synchrotron (Hamburg, Germany) on beamlines BW7A or BW7B with the detectors MAR345 and/or MARCCD at wavelengths of $0.8414 / 0.8874 \AA$ for PBP-A, $1.220 \AA$ for PBP-A-Pen, and $0.8430 \AA$ for PBP-A-L158E and L158E-Pen. Some statistics of data collection and processing are presented in Table 2.

\section{Data processing, structure solution, and refinement}

The diffraction images of the different crystals were processed and merged with the XDS program package. ${ }^{46}$ The crystals are orthorhombic, belong to space group $P 2_{1} 2_{1} 2_{1}$, and are isomorphous, with four molecules in the asymmetric unit. The structure solution was attempted by molecular replacement using the data of the nonacylated PBP-A. A self-rotation function computed at $3.5 \AA$ resolution indicated the presence of a noncrystallographic 4-fold axis along $c$ and, as a consequence, two additional 2-fold axes in the $a$ - $b$ plane, bisecting the two crystallographic axes. A native Patterson function showed a peak of pure translation at $(0.00$, $0.36,0.50)$ occurring because the noncrystallographic 4 -fold axis has the same orientation as a crystallographic 2-fold screw axis. The structure was solved using the program PHASER, ${ }^{47}$ which applies the maximum likelihood to molecular replacement. A set of prealigned class A $\beta$-lactamases was used as a model. Their Protein Data Bank (PDB) codes and the percentage of homology with T.elongatus PBP are as follows: lmfo, 
$32 \%$; 1e25,31\%; 1bsg, 26\%; 1erm, 30\%; 1i2s, 29\%. PHASER succeeded in orienting and locating three molecules in the asymmetric unit consistent with the noncrystallographic 4-fold axis. A fourth molecule was discovered by the program FFFEAR ${ }^{48}$ of the CCP4 suite (1994). ${ }^{49}$ Electron density maps were interpreted using $\mathrm{O}^{50}$ and Coot. ${ }^{51}$ After applying the NCS-phased refinement procedure (CCP4 suite), the refinement was pursued with REFMAC5. ${ }^{52}$ Since all the structures are isomorphous, it was possible to start immediately the refinement of the other structures, after some minor adjustments. Solvent molecules were introduced by ARP/WARP ${ }^{53}$ Ordered glycerol molecules were observed in PBP-A- $\mathrm{L}_{158}$ E and PBP-A-L $\mathrm{L}_{158}$ E-Pen. During the final steps, the hydrogen atoms were incorporated in riding positions and the mean-square displacements of rigid bodies were refined, with each polypeptide chain being defined as a different TLS group. At the end of the refinement, the TLS contribution was included in anisotropic temperature factors. Some refinement statistics are given in Table 2.

\section{Accession codes}

Coordinates and structure factors have been deposited in the PDB: $2 \mathrm{j} 7 \mathrm{v}$ for PBP-A; $2 \mathrm{j} 8 \mathrm{y}$ for PBP-A, acylenzyme complex; 2j9o for PBP-A, L158E mutant; and 2jbf for PBP-A, L158E mutant, acyl-enzyme complex.

\section{Acknowledgements}

This work was supported by grants from the "Fonds National de la Recherche Scientifique" (Belgium), the "Communauté française de Belgique-Action de Recherches Concertées", and the Belgian program of Interuniversity Poles of Attraction initiated by the Federal Office for Scientific Technical and Cultural Affairs (PAI no. P6/19, PROFUSA). We thank Laurence Bausiers for constructing two mutants and Alain Jacques for helping with the structural alignment. We also thank the European Community, Research Infrastructure Action under the FP6 "Structuring the European Research Area Specific Programme" of the European Molecular Biology Laboratory Hamburg Outstation, Contract Number RII3-CT-2004-506008.

\section{Supplementary Data}

Supplementary data associated with this article can be found, in the online version, at doi:10.1016/ j.jmb.2008.12.001

\section{References}

1. Orengo, C. A. \& Thornton, J. M. (2005). Protein families and their evolution-a structural perspective. Annu. Rev. Biochem. 74, 867-900.

2. Gerlt, J. A. \& Babbitt, P. C. (2001). Divergent evolution of enzymatic function: mechanistically diverse super-families and functionally distinct suprafamilies. Annu. Rev. Biochem. 70, 209-246.

3. Joris, B., Ghuysen, J. M., Dive, G., Renard, A., Dideberg, O., Charlier, P. et al. (1988). The active-site-serine penicillin-recognizing enzymes as members of the Streptomyces R61 DD-peptidase family. Biochem. \}. 250, 313-324.

4. Ghuysen, J. M. (1991). Serine beta-lactamases and penicillin-binding proteins. Annu. Rev. Microbiol. $45,37-67$.

5. Massova, I. \& Mobashery, S. (1998). Kinship and diversification of bacterial penicillin-binding proteins and beta-lactamases. Antimicrob. Agents Chemother. 42, 1-17.

6. Ambler, R. P., Coulson, A. F., Frere, J. M., Ghuysen, J. M., Joris, B., Foreman, M. et al. (1991). A standard numbering scheme for the class A beta-lactamases. Biochem. J. 276, 269-270.

7. Matagne, A. \& Frere, J. M. (1995). Contribution of mutant analysis to the understanding of enzyme catalysis - the case of class-A betalactamases. Biochim. Biophys. Acta, 1246,109-127.

8. Adachi, H., Ohta, T. \& Matsuzawa, H. (1991). Site-directed mutants, at position 166, of RTEM-1 beta-lactamase that form a stable acylenzyme intermediate with penicillin, J. Biol. Chem. 266, 3186-3191.

9. Hermann, J. C., Hensen, C., Ridder, L., Mulholland, A. J. \& Holtje, H. D. (2005). Mechanisms of antibiotic resistance: QM/MM modeling of the acylation reaction of a class A beta-lactamase with benzylpenicillin. $f$. Am. Chem. Soc. 127, 4454-4465. 
Published in : Journal of Molecular Biology(2009), vol. 386, pp. 109-120

Status: Postprint (Author's version)

11. Urbach, C., Fastrez, J. \& Soumillion, P. (2008). A new family of cyanobacterial penicillin-binding proteins: a missing link in the evolution of class A beta-lactamases. J. Biol. Chem. 283, 32516-32526.

12. Laskowski, R. A., Macarthur, M. W., Moss, D. S. \& Thornton, J. M. (1993). Procheck-a program to check the stereochemical quality of protein structures, J. Appl. Crystallogr. 26, 283-291.

13. Lovell, S. C., Davis, I. W., Arendall, W. B., de Bakker, P. I. W., Word, J. M., Prisant, M. G. et al. (2003). Structure validation by C alpha geometry: phi, psi and $\mathrm{C}$ beta deviation. Proteins, 50, 437-450.

14. Holm, L. \& Sander, C. (1996). Mapping the protein universe. Science, 273, 595-603.

15. Tranier, S., Bouthors, A. T, Maveyraud, L., Guillet, V., Sougakoff, W. \& Samama, J. P. (2000). The high resolution crystal structure for class A beta-lactamase PER-1 reveals the bases for its increase in breadth of activity, J. Biol. Chem. 275, $28075-28082$.

16. Minasov, G., Wang, X. \& Shoichet, B. K. (2002). An ultrahigh resolution structure of TEM-1 beta-lactamase suggests a role for Glu166 as the general base in acylation. J. Am. Chem. Soc. 124, 5333-5340.

17. Jelsch, C., Mourey, L., Masson, J. M. \& Samama, J. P. (1993). Crystal structure of Escherichia coli TEM1 beta-lactamase at 1.8 A resolution. Proteins, 16, 364-383

18. Ibuka, A. S., Ishii, Y, Galleni, M., Ishiguro, M., Yamaguchi, K., Frere, J. M. et al. (2003). Crystal structure of extended-spectrum betalactamase Toho-1: insights into the molecular mechanism for catalytic reaction and substrate specificity expansion. Biochemistry, 42, 10634 10643

19. Knox, J. R. \& Moews, P. C. (1991). Beta-lactamase of Bacillus licheniformis 749/C-refinement at $2 \AA$ resolution and analysis of hydration, J. Mol. Biol. 220, 435-455.

20. Herzberg, O. (1991). Refined crystal-structure of beta-lactamase from Staphylococcus aureus Pel at 2.0-A resolution, J. Mol. Biol. 217 $701-719$

21. Strynadka, N. C., Adachi, H, Jensen, S. E., Johns, K, Sielecki, A., Betzel, C. et al. (1992). Molecular structure of the acyl-enzyme intermediate in beta-lactam hydrolysis at $1.7 \AA$ resolution. Nature, 359, 700-705.

22. Murphy, B. P. \& Pratt, R. F. (1988). Evidence for an oxyanion hole in serine beta-lactamases and DD-peptidases. Biochem. J. 256, 669672.

23. Fonze, E., Vermeire, M., Nguyen-Disteche, M., Brasseur, R. \& Charlier, P. (1999). The crystal structure of a penicilloyl-serine transferase of intermediate penicillin sensitivity. The DD-transpeptidase of Streptomyces K15. J. Biol. Chem. 274, 21853-21860.

24. Zawadzke, L. E., Chen, C. C. H, Banerjee, S., Li, Z., Wasch, S., Kapadia, G. et al. (1996). Elimination of the hydrolytic water molecule in a class A beta-lactamase mutant: crystal structure and kinetics. Biochemistry, 35, 16475-16482.

25. Lewis, E. R., Winterberg, K. M. \& Fink, A. L. (1997). At mutation leads to altered product specificity in beta-lactamase catalysis. Proc Natl Acad. Sci. USA, 94, 443-447.

26. Chen, C. C. H. \& Herzberg, O. (2001). Structures of the acyl-enzyme complexes of the Staphylococcus aureus beta-lactamase mutant Glul66Asp:Asn170Gln with benzylpenicillin and cephaloridine. Biochemistry, 40, 2351-2358.

27. Escobar, W A., Miller, J. \& Fink, A. L. (1994). Effects of site-specific mutagenesis of tyrosine-105 in a class-A beta-lactamase. Biochem. J. 303, 555-558.

28. Doucet, N., De Wals, P. Y. \& Pelletier, J. N. (2004). Site-saturation mutagenesis of Tyr-105 reveals its importance in substrate stabilization and discrimination in TEM-1 beta-lactamase. J. Biol. Chem. 279,46295^6303.

29. Palzkill, T. \& Botstein, D. (1992). Identification of amino-acid substitutions that alter the substrate-specificity of Tem-1 beta-lactamase J. Bacteriol. 174, 5237-5243.

30. Osuna, J., Viadiu, H, Fink, A. L. \& Soberon, X. (1995). Substitution of Asp for Asn at position-132 in the active-site of Tern betalactamase - activity toward different substrates and effects of neighboring residues, f. Biol. Chem. 270, 775-780.

31. Bouthors, A. T., Dagoneau-Blanchard, N, Naas, T., Nordmann, P., Jarlier, V. \& Sougakoff, W. (1998). Role of residues 104, 164, 166, 238 and 240 in the substrate profile of PER-1 beta-lactamase hydrolysing third-generation cephalosporins. Biochem. $f .330,1443-1449$.

32. Sabbagh, Y., Theriault, E., Sanschagrin, F., Voyer, N., Palzkill, T. \& Levesque, R. C. (1998). Characterization of a PSE-4 mutant with different properties in relation to penicillanic acid sulfones: importance of residues 216 to 218 in class A beta-lactamases. Antimicrob. Agents Chemother. 42, 2319-2325.

33. Pieper, U., Hayakawa, K., Li, Z. \& Herzberg, O. (1997). Circularly permuted beta-lactamase from Staphylococcus aureus PCI. Biochemistry, 36, 8767-8774 
Published in : Journal of Molecular Biology(2009), vol. 386, pp. 109-120

Status: Postprint (Author's version)

34. Osuna, J., Perez-Blancas, A. \& Soberon, X. (2002). Improving a circularly permuted TEM-1 beta-lactamase by directed evolution. Protein Eng. 15, 463-470.

35. Guex, N. \& Peitsch, M. C. (1997). SWISS-MODEL and the Swiss-PdbViewer: an environment for comparative protein modeling. Electrophoresis, 18, 2714-2723.

36. Zafaralla, G., Manavathu, E. K., Lerner, S. A. \& Mobashery, S. (1992). Elucidation of the role of arginine-244 in the turnover processes of class A beta-lactamases. Biochemistry, 31, 3847-3852.

37. Jacob-Dubuisson, E, Lamotte-Brasseur, J., Dideberg, O., Joris, B. \& Frère, J.-M. (1991). Arginine 220 is a critical residue for the catalytic mechanism of the Streptomyces albus G beta-lactamase. Protein Eng. 4, 811-819.

38. Guillaume, G., Vanhove, M., Lamotte-Brasseur, J., Ledent, P., Jamin, M., Joris, B. \& Frere, J. M. (1997). Site-directed mutagenesis of glutamate 166 in two beta-lactamases. Kinetic and molecular modeling studies, J. Biol. Chem. 272, 5438-5444.

39. Gibson, R. M., Christensen, H. \& Waley, S. G. (1990). Site-directed mutagenesis of beta-lactamase I. Single and double mutants of Glu166 and Lys-73. Biochem. 3. 272, 613-619.

40. Damblon, C, Raquet, X., Lian, L. Y., Lamotte-Brasseur, J., Fonze, E., Charlier, P. et al. (1996). The catalytic mechanism of betalactamases: NMR titration of an active-site lysine residue of the TEM-1 enzyme. Proc. Natl Acad. Sci. USA, 93, 1747-1752.

41. Lietz, E. J., Truher, H., Kahn, D., Hokenson, M. J. \& Fink, A. L. (2000). Lysine-73 is involved in the acylation and deacylation of betalactamase. Biochemistry, 39, 4971-4981.

42. Sauvage, E., Kerff, E, Fonze, E., Herman, R., Schoot, B., Marquette, J. P. et al. (2002). The 2.4-A crystal structure of the penicillinresistant penicillin-binding protein PBP5fm from Enterococcus faecium in complex with benzylpenicillin. Cell. Mol. Life Sci. 59,1223-1232.

43. Chesnel, L., Zapun, A., Mouz, N, Dideberg, O. \& Vernet, T. (2002). Increase of the deacylation rate of PBP2x from Streptococcus pneumoniae by single point mutations mimicking the class A beta-lactamases. Eur. J. Biochem. 269, 1678-1683.

44. Dessen, A., Mouz, N, Gordon, E., Hopkins, J. \& Dideberg, O. (2001). Crystal structure of PBP2x from a highly penicillin-resistant Streptococcus pneumoniae clinical isolate: a mosaic framework containing 83 mutations, J. Biol. Chem. 276, 45106-45112.

45. Pettersen, E. E, Goddard, T. D., Huang, C. C, Couch, G. S., Greenblatt, D. M., Meng, E. C. \& Ferrin, T. E. (2004). UCSF Chimera-a visualization system for exploratory research and analysis, J. Comput. Chem. 25, 1605-1612.

46. Kabsch, W. (1993). Automatic processing of rotation diffraction data from crystals of initially unknown symmetry and cell constants, $J$. Appl. Crystallogr. 26, 795-800.

47. Read, R. J. (2001). Pushing the boundaries of molecular replacement with maximum likelihood. Acta Crystallogr., Sect. D: Biol. Crystallogr. 57, 1373-1382.

48. Cowtan, K. (1998). Modified phased translation functions and their application to molecular-fragment location. Acta Crystallogr, Sect. D: Biol. Crystallogr. 54, 750-756.

49. Collaborative Computational Project, No. 4 (1994). The CCP4 suite: programs for protein crystallography. Acta Crystallogr., Sect. D: Biol. Crystallogr. 50, 760-763.

50. Jones, T. A., Zou, J. Y., Cowan, S. W. \& Kjeldgaard, M. (1991). Improved methods for building protein models in electron-density maps and the location of errors in these models. Acta Crystallogr, Sect. A: Found. Crystallogr. 47, 110-119.

51. Emsley, P. \& Cowtan, K. (2004). Coot: model-building tools for molecular graphics. Acta Crystallogr., Sect. D: Biol. Crystallogr. 60 2126-2132.

52. Murshudov, G. N, Vagin, A. A. \& Dodson, E. J. (1997). Refinement of macromolecular structures by the maximum-likelihood method Acta Crystallogr, Sect. D: Biol. Crystallogr. 53, 240-255.

53. Lamzin, V. S. \& Perrakis, A. (2000). Current state of automated crystallographic data analysis. Nat. Struct. Biol. 7, 978-981.

54. Konagurthu, A. S., Whisstock, J. C, Stuckey, P. J. \& Lesk, A. M. (2006). MUSTANG: a multiple structural alignment algorithm. Proteins, 64, 559-574.

55. Hall, T. A. (1999). BioEdit a user-friendly biological sequence alignment editor and analysis program for Windows $95 / 98 / \mathrm{NT}$ Nucleic Acids Symp. Ser. 41, 95-98.

56. Silvaggi, N. R., Josephine, H. R., Kuzin, A. P., Nagarajan, R., Pratt, R. F. \& Kelly, J. A. (2005). Crystal structures of complexes between the R61 DD-pepti-dase and peptidoglycan-mimetic beta-lactams: a non-covalent complex with a "perfect penicillin", $f$. Mol. Biol. $345,521-533$. 